\title{
BISE BEPORT
}

\section{HEPATIC RESECTION FOR BILE DUCT INJURY: A CASE REPORT Utsav Joshi' ${ }^{1}$ Ramesh Singh Bhandari ${ }^{1 *}$}

\footnotetext{
${ }^{1}$ Department of Surgery, Department of GI and General Surgery, T U Teaching Hospital, Kathmandu, Nepal
}

*Correspondence to: Dr Ramesh Singh Bhandari, Department of Gl and General Surgery, TUTH, Kathmandu, Nepal. Email: rsbhandariO9@gmail.com

\begin{abstract}
Background: Bile duct injury is a serious complication following both open and laparoscopic cholecystectomy. The extent of injury may be severe enough to consider biliary reconstruction procedures like Roux-en-Y hepaticojejunostomy or even hepatectomyinrareinstancesforthedefinitivemanagement.Case:A56yearoldfemale, whounderwentopencholecystectomy and detected bile duct injury intraoperatively. Small feeding tube was placed in the bile duct, exteriorized and patient was referred to our center for further management. Liver function tests at presentation revealed cholestatic patterns of liver derangement but the patient did not show any signs suggestive of sepsis. Endoscopic Retrograde Cholangiopancreatography revealed complete stricture of common hepatic duct. Magnetic Resonance Cholangiopancreatography revealed Bismuth type 4 bile duct stricture. The plan was to perform a bilateral hepaticojejunostomy, however, because of the very difficult anatomy and failure to identify the right duct, right hepatectomy with left duct hepaticojejunostomy was performed as a definitive management for her type IV bile duct injury. The patient had an uneventful post-operative course. Conclusion: Infrequently, liver resection remains an important therapeutic option in cases of complicated and major forms of bile duct injuries where the bilateral biliary reconstruction is not feasible.
\end{abstract}

Key words: Bile duct injury, Cholecystectomy, Hepatectomy.

\section{INTRODUCTION}

Bile duct injury (BDI) is one of the major complications of both open and laparoscopic cholecystectomies. Incorrect identification or misinterpretation of the biliary anatomy along with dangerous pathology and surgery predispose to bile duct injuries during cholecystectomies. ${ }^{1}$

Management of bile ductinjuries includes endoscopic and percutaneous procedures, hepaticojejunostomy and rarely hepatectomy. Minor bile duct injuries may be managed endoscopically but major complex injuries may require biliary reconstruction procedures based on the degree of vascular injury, liver necrosis, abscess formation, acute liver failure or biliary cirrhosis. ${ }^{2}$ Even though liver resection is uncommon and a last resort option, 5.6\%- $15 \%$ of cases of major bile duct injuries require liver resection as a definite procedure. ${ }^{3}$
We present an interesting case of grade IV bile duct injury sustained during open cholecystectomy that was managed with right hepatectomy and hepaticojejunostomy.

\section{Case presentation}

A 56 year old female with a history of symptomatic Cholelithiasis for past 8 to 10 years underwent open cholecystectomy in a rural hospital. Common bile duct (CBD) injury was detected intraoperatively. Proximal visible portion of the bile duct was cannulated with a feeding tube and which was then exteriorized. A subhepatic drain was also placed and the abdomen closed. Postoperatively, the pain abdomen persisted and there was continuous drainage of bilious fluid from the abdominal drain. After persistent deterioration of general condition of the patient, she was referred to our department 17 
days later. At presentation, she had persistent right upper quadrant pain without fever. Her laboratory parameters revealed: Hemoglobin: 8.5 gram\%, White blood cells: $5500 / \mathrm{mm}^{3}$ (Neutrophils: $56 \%$, Lymphocytes: 38\%, Monocytes: 4\%, Eosinophils: 2\%), Platelets: $516000 / \mathrm{mm}^{3}$, PT: 15 seconds. Her renal function tests were normal (Urea: $5.3 \mathrm{mmol} / \mathrm{l}$, Creatinine: $60 \mu \mathrm{mol} / \mathrm{l})$. The liver function tests revealed cholestatic pattern of liver derangement (Total bilirubin: $75 \mu \mathrm{mol} / \mathrm{l}$, Direct bilirubin: 46 $\mu \mathrm{mol} / \mathrm{I}$, Alkaline phosphatase: $333 \mathrm{U} / \mathrm{L}$, Aspartate transaminase: $61 \mathrm{U} / \mathrm{L}$, Alanine transaminase: 84 $\mathrm{U} / \mathrm{L}$ ). The abdominal drain revealed $150 \mathrm{ml}$ of bilious fluid. Bile from the abdominal drain was cultured which showed no growth in 48 hours of incubation. The feeding tube had already been dislodged.

The patient was planned for Endoscopic Retrograde Cholangiopancreatography (ERCP) on $21^{\text {st }}$ postoperative day which revealed complete stricture of common hepatic duct with no visualization of the right and left intrahepatic biliary ducts (IHBD). Magnetic Resonance Cholangiopancreatography (MRCP) (Figure 1) was done on $27^{\text {th }}$ post-operative day which revealed Bismuth type 4 of bile duct injury. The patient was continued on conservative treatment and planned for a definitive procedure

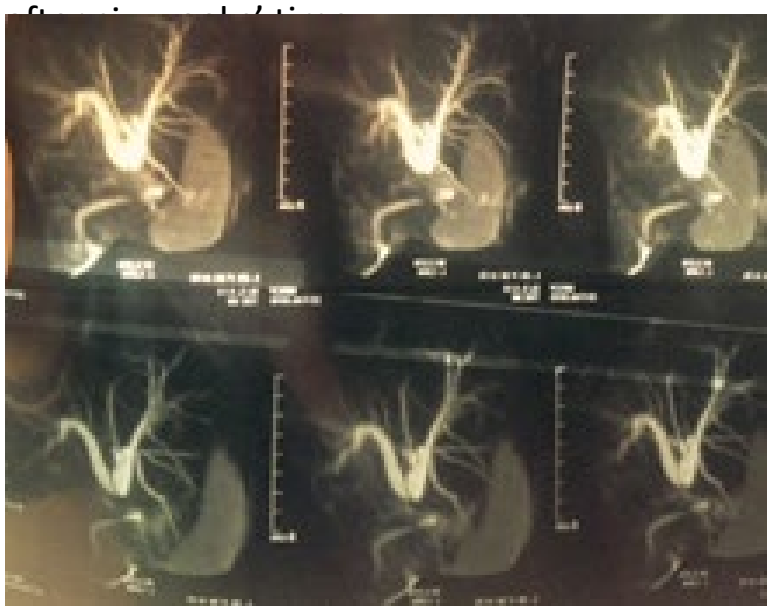

Figure 1. MRCP Showing Bismuth Type 4 Bile duct injury

The surgical management included right subcostal laparotomy with a reverse $L$ incision. The right hepatic duct could not be dissected due to densely adhered hilum. The left hepatic duct was dissected and isolated. Henceforth, right hepatectomy was done by Kelly clamp method using cautery. The jejunum was then, transected, distal part was mobilized retrocolically and a Roux-en-Y hepaticojejunostomy (Figure 2) was performed. Her post-operative course was uneventful except few episodes of vomiting on fifth post-operative day. The patient was discharged 7 days after surgery. At six months follow up, patient has been doing well with normal liver function test.

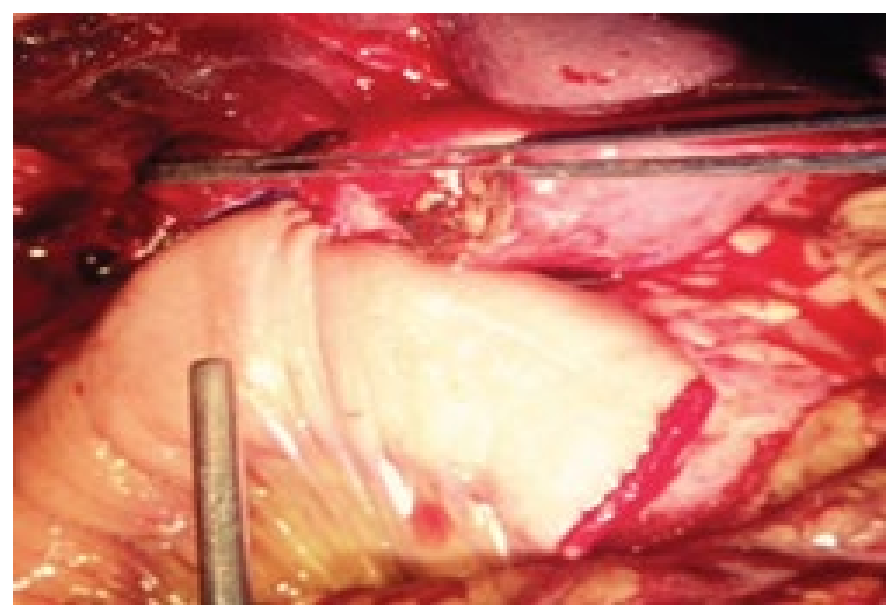

Figure 2. Completed Hepaticojejunostomy to the left duct

\section{DISCUSSION}

This case report describes an atypical case of postcholecystectomy bile duct injury that was managed with hepatic resection and bilioenteric anastomosis.

latrogenic injury to the biliary tract is one of the most important complication of cholecystectomy. Despite technical and surgical advances and cholecystectomy being one of the most common operative procedure performed nowadays, bile duct injuries still has an incidence of $0.2 \%-0.3 \%$ in patients undergoing an open procedure and $0.5 \%-0.8 \%$ in those undergoing laparoscopic cholecystectomy. ${ }^{4}$ Around, $70-80 \%$ of all bile duct injuries occur due to incorrect identification and interpretation of the biliary anatomy during open or laparoscopic procedures. ${ }^{5}$ Misidentification or misinterpretation may be even more exaggerated due to anatomical anomalies of the biliary tracts. Cystic duct that runs along the side of a low lying aberrant right segmental duct may predispose to biliary injuries. ${ }^{2}$ Anomalous insertion of cystic duct into common hepatic duct may result in misidentification of bile duct as cystic duct. ${ }^{5}$ Hence, proper identification and careful dissection around the neck of gallbladder is the best way to avoid these injuries. 
Patients with bile duct injury who have persistent bile leakage may develop biloma or abscess. Such patients may develop severe pain abdomen, fever with chills and additional signs of sepsis. On the other hand, certain patients may develop strictures secondary to bile duct injury and such patients may develop cholestatic jaundice as their predominant presentation. Abdominal ultrasonography helps us to visualize intra and extrahepatic bile ducts along with any subhepatic collections in case of bile leaks. With ERCP, biliary tracts can be evaluated distal to the level of injury. MRCP delineates the biliary anatomy both proximal and distal to the level of injury and also facilitates identification of fluid collections. Early diagnosis of bile duct injuries is of utmost importance since failure to recognize may result in serious complications like biliary cirrhosis, liver failure and death. ${ }^{6}$

Multiple approaches have been proposed to achieve a definite management of post-cholecystectomy bile duct injury: endoscopic, percutaneous, bilioenteric anastomosis, liver resection and transplantation. ${ }^{3}$ Minor bile duct injuries may simply be managed with ERCP with sphincterotomy or stent placement. Major bile duct injuries, on the other hand, require major biliary reconstruction procedures for definite management; Roux-en-Y hepaticojejunostomy being the commonest procedure. ${ }^{7}$

Liver resection for the management of bile duct injuries is uncommon. Despite a large number of patients who sustain bile duct injury following cholecystectomy, only a few cases have been reported in literature who underwent major hepatectomy. Pekolj et al. reported an estimated $5 \%$ of the patients requiring a major hepatectomy following bile duct injury in a study including 287 patients. ${ }^{3}$ Study by Li et al. reports $13.2 \%$ of the patients with iatrogenic bile duct injury, either as an isolated damage or in combination with vascular injury, underwent hepatectomy. ${ }^{8}$ In fact, hepatectomy is considered as a last resort option in cases of liver necrosis secondary to major vascular injury, unreconstructable hepatic ducts, multiple failed repairs and repeated cholangitis. ${ }^{6}$ According to Truant et al, proximal location of biliary injuries with disruption of hepatic ductal confluence and/ or concurrent arterial injuries are major indications for hepatectomy. ${ }^{9}$ This was also the case in our patient who had sustained injury in the region of hepatic ductal confluence. However, uncommon though it may be, hepatectomy can lead to good long term results in $72 \%-100 \%$ of patients; results which are comparable to other procedures like hepaticojejunostomy. ${ }^{4}$

In the presented case, the patient was initially planned for bilateral hepaticojejunostomy. During the procedure, complete transection of right anterior sectoral duct and left hepatic duct with dense adhesions in the hilum was noted. The right hepatic duct however, could not be dissected due to the dense hilar adhesions and thus, hepaticojejunostomy was not technically feasible on the right side. For this reason, right hemihepatectomy was performed with subsequent hepaticojejunostomy on the left side. In this case, without hepatectomy, there were chances that the patient might continue to develop recurrent cholangitis due to the stenosed right hepatic duct in the future. So, right hepatectomy with hepaticojejunostomy was the best treatment option for this patient.

\section{CONCLUSION}

Since cholecystectomies are one of the most common operative procedures performed, the incidence of bile duct injuries are sure to rise. It is imperative to correctly identify and interpret the biliary anatomy before ligation or transection of structures during surgery to avoid chances of bile duct injury. Once bile duct injuries occur, early diagnosis, prompt referral and appropriate management is of utmost importance. Even though indications for hepatectomy are limited, liver resection should always be considered as an important therapeutic option in cases of complicated and major forms of bile duct injuries.

\section{REFERENCES:}

1. Wojcicki M, Patkowski W, Chmurowicz T, Bialek A, Wiechowska-Kozlowska A, Stankiewicz $R$, Milkiewicz $P$, Krawczyk $M$. Isolated right posterior bile duct injury following cholecystectomy: Report of two cases. World journal of gastroenterology: WJG 2013 Sep 28;19(36):6118.

2. Jadrijevic S, Sef D, Kocman B, Mrzljak A, Matasic H, Skegro D. Right hepatectomy due to JCMC/ Vol 7/ No. 1/ Issue 19/ Jan-Mar, 2017 
portal vein thrombosis in vasculobiliary injury following laparoscopic cholecystectomy: a case report. Journal of medical case reports 2014 Dec $7 ; 8(1): 1$.

3. Pekolj J, Yanzón A, Dietrich A, Del Valle G, Ardiles $V$, de Santibanes E. Major liver resection as definitive treatment in post-cholecystectomy common bile duct injuries. World journal of surgery 2015 May 1;39(5):1216-23.

4. Perini MV, Herman $P$, Montagnini $A L$, Jukemura J, Coelho FF, Kruger JA, Bacchella T, Cecconello I. Liver resection for the treatment of postcholecystectomy biliary stricture with vascular injury. World Journal of Gastroenterology: WJG 2015 Feb 21;21(7):2102.

5. Jabłońska B, Lampe P. latrogenic bile duct injuries: etiology, diagnosis and management. World J Gastroenterol 2009 Sep 7;15(33):4097104.
6. Jabłońska B. Hepatectomy for bile duct injuries: when is it necessary?. World J Gastroenterol 2013 Oct 14;19(38):6348-52.

7. Hajjar NA, Tomuş C, Mocan L, Mocan T, Graur F, lancu C, Zaharie F. Management of bile duct injuries following laparoscopic cholecystectomy: long-term outcome and risk factors infuencing biliary reconstruction. Chirurgia (Bucharest, Romania: 1990). 2013 Dec;109(4):493-9.

8. Li J, Frilling A, Nadalin S, Broelsch CE, Malago $M$. Timing and risk factors of hepatectomy in the management of complications following laparoscopic cholecystectomy. Journal of gastrointestinal surgery 2012 Apr 1;16(4):81520.

9. Truant S, Boleslawski E, Lebuffe G, Sergent G, Pruvot FR. Hepatic resection for postcholecystectomy bile duct injuries: a literature review. HPB 2010 Jun 1;12(5):334-41. 